\title{
Birth Control Method
}

National Cancer Institute

\section{Source}

National Cancer Institute. Birth Control Method. NCI Thesaurus. Code C106501.

The method by which conception or impregnation is prevented. This may be achieved through the use of devices, drugs or surgery. 